\title{
PAPR Reduction in LFDMA Using Hadamard Transform Technique
}

\author{
Mohamed M. El-Nabawy ${ }^{1}$, Mohamed A. Aboul-Dahab ${ }^{2}$, Khairy El-Barbary ${ }^{3}$ \\ ${ }^{1}$ Modern Academy for Eng. \& Tech in Maadi (M.A.M)/ Electronic and Communication Dept., Cairo, Egypt \\ ${ }^{2}$ Arab Academy for Science and Technology and Maritime Transport (AAST)/ Electronic and Communication Dept., Cairo, Egypt \\ ${ }^{3}$ Canal University, Electronic and Communication Dept., Cairo, Egypt
}

\section{Email address:}

eng.mohamed.elnabawy@gmail.com (M. M. El-Nabawy),mdahab@aast.edu (M. A. Aboul-Dahab), khbar2000@yahoo.com (K. El-Barbary)

To cite this article:

Mohamed M. El-Nabawy, Mohamed A. Aboul-Dahab, Khairy El-Barbary. Papr Reduction in Lfdma Using Hadamard Transform Technique. International Journal of Wireless Communications and Mobile Computing. Vol. 3, No. 4, 2015, pp. 40-45.

doi: 10.11648/j.wcmc.20150304.11

\begin{abstract}
The power consumption is an essential issue for designers of mobile devices. Orthogonal Frequency Division Multiplexing (OFDM) suffers from its Peak - to - Average Power Ratio (PAPR) problem. The Discrete Fourier Transmission Spread OFDM (DFTS-OFDM) based on Single Carrier Frequency Division Multiple Access (SC-FDMA) has been widely adopted due to its lower peak-to-average power ratio (PAPR) of transmits signals compared with OFDM. There are three types of mapping in SCFDMA. These namely Localized FDMA (LFDMA), Distributed FDMA (DFDMA), and Interleaved FDMA (IFDMA). In this paper, we propose a modified SCFDMA. This is based on using Hadamard transform instead of Fast Fourier Transform (FFT). Utilizing LFDMA Mapping techniques this is proposed for uplink scenario of LTE. Simulation results show that the proposed scheme reduces PAPR compared to the conventional SCFDMA while the BER does not degraded.
\end{abstract}

Keywords: Orthogonal Frequency Division Multiplexing (OFDM), Single Carrier Frequency Division Multiple Access (SCFDMA), Localized FDMA (LFDMA), Distributed FDMA (DFMA), Interleaved FDMA (IFDMA), Cumulative Complementary Distribution Function (CCDF), Peak to Average Power Ratio (PAPR), Bit Error Rate (BER)

\section{Introduction}

Broadband wireless communication systems are designed to provide high-data-rate services to satisfy the increasing demands of the future wireless networks. As the bit rate increases, the problem of inter-symbol interference becomes more serious. Orthogonal frequency division multiple access (OFDMA) is an attractive technology to deal with the detrimental effects of multipath fading, but it has several inherent drawbacks such as the large peak-to-average-power ratio (PAPR) and the sensitivity to carrier frequency offsets $[1,2]$. Recently, much attention has been focused on another broadband wireless communication system, which is the single carrier frequency division multiple access (SC-FDMA) system $[3,4]$.

The SC-FDMA has two main advantages over orthogonal frequency division multiplexing (OFDM), namely, a lower PAPR and a lower sensitivity to carrier frequency errors [3].
There are two methods to choose the subcarriers in SCFDMA systems: interleaved subcarrier mapping and localized subcarrier mapping. We will refer to the localized subcarrier mapping mode of SC-FDMA as localized frequency division multiple access (LFDMA) and the interleaved subcarrier mapping mode as interleaved frequency division multiple access (IFDMA). The LFDMA system incurs a higher PAPR compared to the IFDMA system but, compared to OFDM; it is lower, though not significantly [3]. Thus, we will be concerned with the LFDMA system only.

For OFDM systems, PAPR reduction techniques have been introduced to reduce problems at the high-power amplifier and/or alleviate its back-off specifications by companding or clipping the amplitude of the OFDM signals [5-9].

In this paper, we will discuss and evaluate the PAPR of a modified transceiver scheme of SCFDMA. The proposed system is similar to SCFDMA except that it uses Hadamard Transform and Inverse Hadamard Transform instead of DFT, 
FFT, IDFT, and IFFT. This paper is organized as follows: In section II presents the SCFDMA system model and the concept PAPR problem. In section III the proposed system is discussed. Simulation results are reported in section IV and conclusions are presented in V.

\section{SC-FDMA System Model}

Fig. 1 shows the block diagram of a basic LTE SC-FDMA system. SC-FDMA is a modified structure of OFDMA system [8]. SC-FDMA can be viewed as Discrete Fourier Transform (DFT)-spread OFDMA or frequency-spread OFDMA, where time domain data symbols are transformed to frequency domain by DFT before going through OFDMA system [9].

The input to the SC-FDMA transmitter is a stream of $\mathrm{x}$ bits that is converted to multilevel sequences of complex number $\mathrm{x}(\mathrm{s})$, where $\mathrm{x}(\mathrm{s})$ is the data symbol, and $\mathrm{s}$ is the sample index. Then, the transmitter concatenates the modulation symbols into blocks through serial to parallel converter $(\mathrm{S} / \mathrm{P})$ block, each containing $\mathrm{M}$ symbols. These modulated symbols perform M-point DFT to produce a frequency domain representation as follows:

$$
\begin{aligned}
& X(k)=\sum_{m=0}^{M-1} x(n) e^{\frac{-j 2 \Pi m k}{M}} \\
& \begin{array}{l}
\text { Binary } \\
\text { inputs }
\end{array} \\
& \longrightarrow \text { Mapper }
\end{aligned}
$$

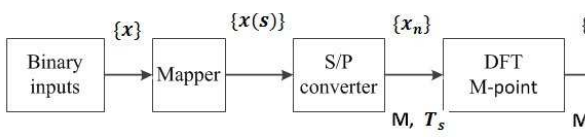

The output of the DFT is then applied to a subcarrier mapping block which maps each of the M-DFT outputs to one of the $\mathrm{N}$ orthogonal subcarriers $(\mathrm{N}>\mathrm{M})$ that can be transmitted. The ratio between $\mathrm{N}$ and $\mathrm{M}$ is called the bandwidth expansion factor of the symbol sequence or the spreading factor $\mathrm{Q}$ $(\mathrm{Q}=\mathrm{N} / \mathrm{M})$ [4]. If all terminals transmit $\mathrm{M}$ symbols per block, the system can handle Q simultaneous transmissions without co-channel interference. The outcome of the subcarrier mapping is $\mathrm{N}$ of complex subcarrier amplitudes $\breve{\mathrm{X}}(\mathrm{l})(1=0,1$, $2 \ldots, \mathrm{N}-1)$, The outputs of subcarrier mapping block are used for N-point Inverse Fast Fourier Transform (N-IFFT) which transforms the $N$ subcarriers to a signal $\breve{x}(m)$ in time domain, then each $\breve{x}(m)$ is transmitted sequentially.

In DFT spreading technique, the allocation of subcarriers to users is done using one of the most schemes, Localized FDMA (LFDMA), Distributed FDMA (DFDMA) and Interleaved FDMA (IFDMA). In LFDMA scheme, M consecutive subcarriers are allocated in a total of $\mathrm{N}$ subcarriers and the consecutive unused subcarriers are filled with zeroes. DFDMA scheme involves allocation of $\mathrm{M}$ outputs over the entire band of $\mathrm{N}$ subcarriers and the remaining unused subcarriers are filled with zeroes. IFDMA scheme involves allocation of M DFT outputs over total of $\mathrm{N}$ subcarriers with equidistance.

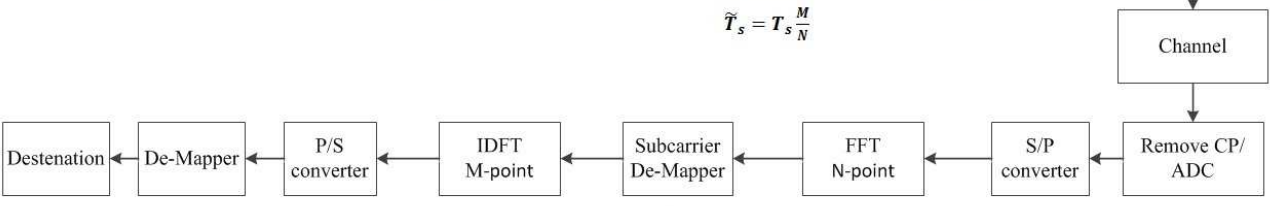

M number of assigned subcarriers, $\mathrm{N}$ number of available subcarriers, and $T_{s}, \tilde{T}_{s}$ symbol duration

Figure 1. Basic block diagram of SC-FDMA

The allocation of subcarriers using the three schemes is shown in Fig (2). The total number of subcarriers is $\mathrm{N}=12$. Here we assume that 4 DFT outputs are distributed $(M=4)$. The spreading factor is $\mathrm{Q}=3$. $\mathrm{X}(0), \mathrm{X}(1), \mathrm{X}(2), \mathrm{X}(3)$ represent the DFT outputs that are distributed using LFDMA, DFDMA and IFDMA schemes. In the following sections, we derive the IFFT outputs for LFDMA scheme. The IFFT output in each scheme is obtained after the DFT and subcarrier mapping operations. The significance of IFFT outputs is that it gives the information about the time domain signal depending on certain factors like scaling, phase rotation, complex weighting factor, etc.

\begin{tabular}{|c|c|c|c|c|c|c|c|c|c|c|c|}
\hline $\mathbf{X}(0)$ & $\mathbf{X}(\mathbf{1})$ & $\mathbf{X}(2)$ & $\mathbf{X}(3)$ & 0 & 0 & 0 & 0 & 0 & 0 & 0 & 0 \\
\hline \multicolumn{12}{|c|}{ (a) LFDMA } \\
\hline $\mathbf{X}(0)$ & 0 & $\mathbf{X}(\mathbf{1})$ & 0 & $\mathbf{X}(2)$ & 0 & $\mathbf{X}(3)$ & 0 & 0 & 0 & 0 & 0 \\
\hline \multicolumn{12}{|c|}{ (b) DFDMA } \\
\hline $\mathbf{X}(0)$ & 0 & 0 & $\mathbf{X}(\mathbf{1})$ & 0 & 0 & $\mathbf{X}(2)$ & 0 & 0 & $\mathbf{X}(\mathbf{3})$ & 0 & 0 \\
\hline
\end{tabular}

Figure 2. DFT Spreading Technique showing distribution of DFT outputs $(N=12, M=4, Q=3)$.

\subsection{IFFT Output for LFDMA}

Let us assume a total number of $\mathrm{N}$ subcarriers and $\mathrm{M}$ be the consecutive DFT outputs that are allocated with the unused subcarriers filled with zeroes.

Let us define $\mathrm{n}=\mathrm{Qm}+\mathrm{l}$ where $\mathrm{Q}$ is the Spreading factor where $0 \leq \mathrm{l} \leq \mathrm{L}-1$ and $0 \leq \mathrm{m} \leq \mathrm{M}-1$.

The output if SC-LFDMA after the subcarrier mapping is

$$
X_{L F D}(k)= \begin{cases}X(k), & k=0,1, \ldots . .,(M-1) \\ 0, & \text { otherwise }\end{cases}
$$

so that the IFFT output sequence is given by

$$
x_{L F D}(n)=x_{L F D}(Q m+1), 0 \leq \mathrm{n} \leq \mathrm{N}-1
$$

Now 


$$
\begin{gathered}
x_{L F D}(n)=\frac{1}{N} \sum_{k=0}^{N-1} X_{L F D}(k) e^{\frac{j 2 \pi k n}{N}} \\
\therefore x_{L F D}(n)=\frac{1}{Q M} \sum_{k=0}^{M-1} X(k) e^{\frac{j 2 \pi k(Q m+l)}{Q M}}
\end{gathered}
$$

If $\mathrm{l}=0$, then from $(5)$

$$
\begin{gathered}
\therefore x_{L F D}(n)=\frac{1}{Q M} \sum_{k=0}^{M-1} X(k) e^{\frac{j 2 \pi k m}{M}} \\
\therefore x_{L F D}(n)=\frac{1}{Q} x(m)
\end{gathered}
$$

From (6) It can be seen that the IFFT output for LFDMA depends on the scaled version of the input signal by $1 / \mathrm{Q}$.

\subsection{Concept of PAPR}

Peak to Average Power Ratio (PAPR) occurs due to the summing of carriers together. The maximum peak power increases proportionally to the number of carriers in the system. After linear region, the scalar relationship is lost and the amplifier moves into saturation region. The use of amplifiers in the saturation region leads to distortion which is a major drawback.

PAPR is expressed as

$$
P A P R=\frac{\substack{N-1 \\ \sum=0}}{P_{a v}\{x(n)\}}
$$

Where $x(n)$ is the original signal, $\max \left\{x^{2}(n)\right\}$ Indicates the peak signal power, $\mathrm{N}$ is the total number of data symbols and

$$
P_{a v}\{x(n)\}=\frac{1}{N} \sum_{n=0}^{N-1} E\left\{x^{2}(n)\right\}
$$

indicates the average signal power $E\left\{x^{2}(n)\right\}$ in (8) is the mean square value of $\mathrm{x}(\mathrm{n})$ and ' $\mathrm{E}$ ' stands for expectation.

\section{The Proposed Scheme}

A baseband model of proposed system is shown in Figure 3. The proposed system is similar to SCFDMA except that it uses Hadamard Transform and Inverse Hadamard Transform instead of DFT, FFT, IDFT, and IFFT.

\subsection{Hadamard Transform}

The proposed scheme is based mainly on the use of hadamard transform replaced of FFT module. Park et.al; have proposed in [10] a scheme for PAPR reduction in OFDM transmission using hadamard transform. The proposed hadamard transform scheme may reduce the occurrence of the high peaks comparing the original OFDM system. The idea to use the hadamard transform is to reduce the autocorrelation of the input sequence to reduce the peak to average power problem and it requires no side information to be transmitted to the receiver. In the section, we briefly review hadamard transform. We assume $\mathrm{H}$ is the hadamard transform matrix of $\mathrm{N}$ orders, and hadamard matrix is standard orthogonal matrix. Every element of hadamard matrix only is 1 or -1 . The hadamard matrix of 2 orders is stated by

$$
H_{2}=\frac{1}{\sqrt{2}}\left(\begin{array}{cc}
1 & 1 \\
1 & -1
\end{array}\right)
$$

hadamard matrix of $2 \mathrm{~N}$ order may be constructed by

$$
H_{2 N}=\frac{1}{\sqrt{2 N}}\left(\begin{array}{cc}
H_{N} & H_{N} \\
H_{N} & -H_{N}
\end{array}\right)
$$

Where $-H_{N}$ is the complementary of $H_{N}$. Hadamard matrix satisfy the relation

$$
H_{2 N} H_{2 N}^{T}=H_{2 N}^{T} H_{2 N}=I_{2 N}
$$

Where $H_{2 N}^{T}$ is the transport matrix, $I_{2 N}$ is the unity matrix of $2 \mathrm{~N}$ order. Note that Hadamard transform is an orthogonal linear transform and can be implemented by a butterfly structure as in FFT. This means that applying Hadamard transform does not require the extensive increase of system complexity.

After the sequence $x=\left[\begin{array}{llll}x_{1} & x_{2} \ldots \ldots x_{N}\end{array}\right]^{T}$ is transformed by hadamard matrix of $\mathrm{N}$ order, the new sequence is

$$
Y=H X
$$

\subsection{Description of Proposed Scheme}

$\mathrm{M}$ is the number of subcarriers assigned to each user. $\mathrm{N}$ is the total number of available subcarriers. $\mathrm{N}=\mathrm{Q} . \mathrm{M}$, where $\mathrm{Q}$ is the spreading factor and $\mathrm{N}>\mathrm{M}$. Hadamard transform performs an orthogonal, symmetric, involution, linear operation on $2 \mathrm{~m}$ real numbers (or complex numbers) which can be generated by, $\mathrm{HO}=1$ and then define for $\mathrm{m}>0$. The Hadamard transform is $2^{\mathrm{m}} \mathrm{X} 2^{\mathrm{m}}$ matrix.

$$
H_{m}=\frac{1}{\sqrt{2}}\left(\begin{array}{cc}
H_{m-1} & H_{m-1} \\
H_{m-1} & -H_{m-1}
\end{array}\right)
$$

Compared to the Fast Fourier Transform (FFT), the Hadamard transform requires less storage space and is faster to calculate because it uses only real additions and subtractions, while the FFT requires complex values.

We choose Localized Frequency Division Multiple Access (LFDMA) subcarrier mapping mode. An example of Hadamard SCFDMA transmit symbols for $\mathrm{M}=4, \mathrm{Q}=3, \mathrm{~N}=$ 
12 is illustrated in Figure 4. $x_{n}$ are the transmit symbols in time domain passed through Hadamard transform to get $x_{k}$ which are time domain samples. Therefore, subcarrier mapping is done in time domain. After the subcarrier mapping is done, the data is passed through inverse Hadamard transform.

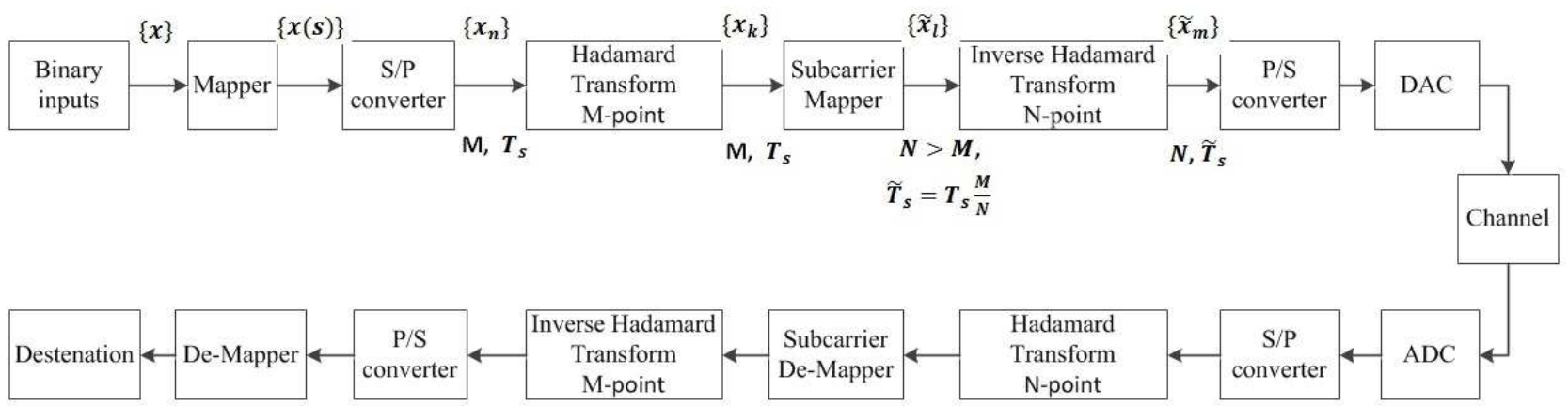

M number of assigned subcarriers, $\mathrm{N}$ number of available subcarriers, and $T_{S}, \tilde{T}_{s}$ symbol duration

Figure 3. Block diagram of proposed SC-FDMA system

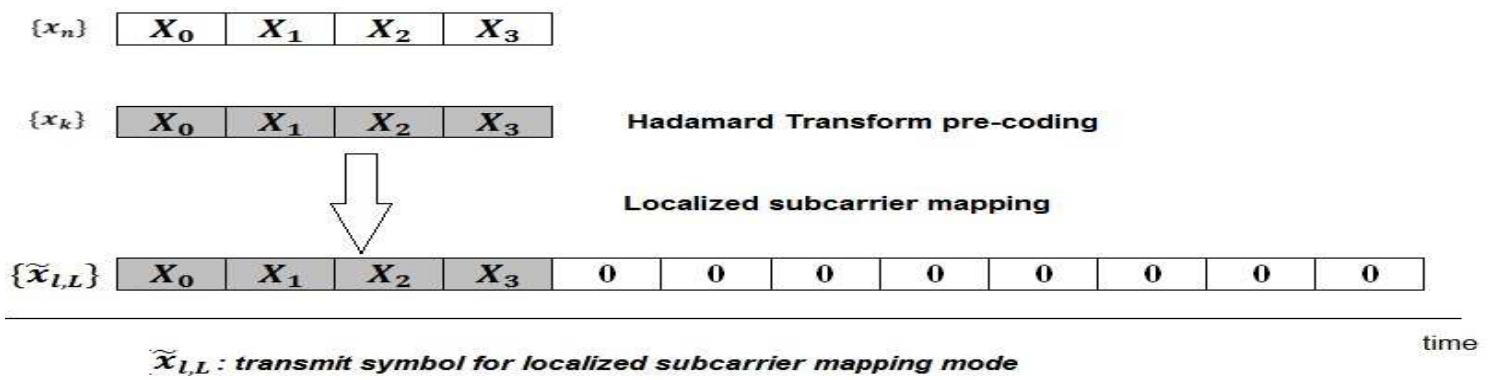

Figure 4. An example of Hadamard SCFDMA transmit symbol for $N=4, Q=3$ and $M=12$.

We analyze the PAPR of Hadamard SCFDMA signal with companding technique for LFDMA subcarrier mapping mode. In the subsequent derivations, we will assume $\mathrm{N}=\mathrm{Q} . \mathrm{M}$ and follow the notations in Figure 3. Let the data block of $M$ complex valued symbols to be modulated is $\left\{x_{n}: \mathrm{n}=0, \ldots, \mathrm{M}-1\right\}$.

Then, by applying M-point Hadamard transform to, $x_{n}$, $\left\{x_{k}: \mathrm{k}=0, \ldots, \mathrm{M}-1\right\}$ the time domain symbols can be described as

$$
x_{k}=H_{N} x_{n}^{T} ; \quad \mathrm{k}=0, \ldots \ldots . ., \mathrm{N}-1
$$

$H_{N}$ is the Hadamard matrix and can be obtained from (13). $\left\{\tilde{\mathrm{x}}_{\mathrm{l}}: \mathrm{l}=0, \ldots \ldots, \mathrm{N}-1\right\}$ are time domain samples after subcarrier mapping. For IFDMA (subcarrier mapping technique) the time domain samples $\tilde{\mathrm{x}}_{1}$ after mapping can be represented as

$$
\tilde{\mathrm{x}}_{\mathrm{l}}= \begin{cases}\mathrm{X}_{\mathrm{l} / \mathrm{Q}} \quad \mathrm{l}=\mathrm{Q} . \mathrm{k} & \mathrm{k}=0, \ldots \ldots, \mathrm{M}-1 \\ 0 \quad \text { otherwise } & \mathrm{l}=0, \ldots \ldots, \mathrm{N}-1\end{cases}
$$

$\left\{\tilde{x}_{m}\right\}$ are time domain symbols after inverse Hadamard transform of $\left\{\tilde{x}_{1}\right\}$ given by

$$
\tilde{\mathrm{x}}_{\mathrm{m}}=\operatorname{inv}\left(\mathrm{H}_{\mathrm{N}}\right) \cdot \tilde{\mathrm{x}}_{\mathrm{l}} \quad ; \mathrm{m}, \mathrm{l}=0, \ldots \ldots, \mathrm{N}-1
$$

where $H_{N}$ is the Hadamard matrix and can be obtained from (13).
The PAPR is the measure of the peak-to-average power ratio. Battery operated terminal must have low PAPR when transmitting a signal so as the power amplifier operates in its linear region.

The PAPR of the transmitted signal can be expressed as:

$$
C C D F\left(N, P A P R_{0}\right)=\operatorname{Pr}\left\{P A P R>P A P R_{0}\right\}=1-\left(1-P A P R_{0}\right)^{N}
$$

Where $\mathrm{N}$ is the number of subcarriers and $P A P R_{0}$ is a certain value of PAPR.

\section{Simulation Results}

To show the overall effect of the proposed scheme on reducing PAPR, we consider 500000 Number of iteration blocks to calculate the CCDF (Complementary Cumulative Distribution Function) of the PAPR. CCDF of PAPR for SCFDMA, Hadamard SCFDMA and OFDMA are evaluated and compared. The subcarrier mapping is LFDMA. In the simulations, the total number of subcarriers $\mathrm{N}$ is set to 256 ; input data block size $\mathrm{M}$ to 64 , the spreading factor $\mathrm{Q}$ to 4 , and compered CCDF of PAPR at Q-PSK, 16-QAM, and 64QAM mapper.

In figure 5, The SCFDMA system incurs a lower PAPR compared to OFDM, and the performance of the proposed scheme provides better performance than classical SCFDMA.

We can see in figure 5(a) the PAPR of proposed scheme is almost $0 \mathrm{~dB}$, which mean the probability to occur the PAPR problem when used Q-PSK QAM is equal zero, from the 
same figure we can ensure that the PAPR of SC-FDMA is

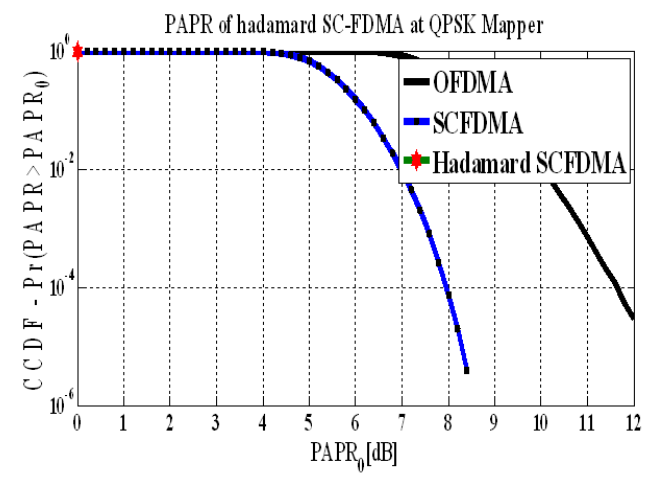

(a) With Q-PSK less than OFDM system.

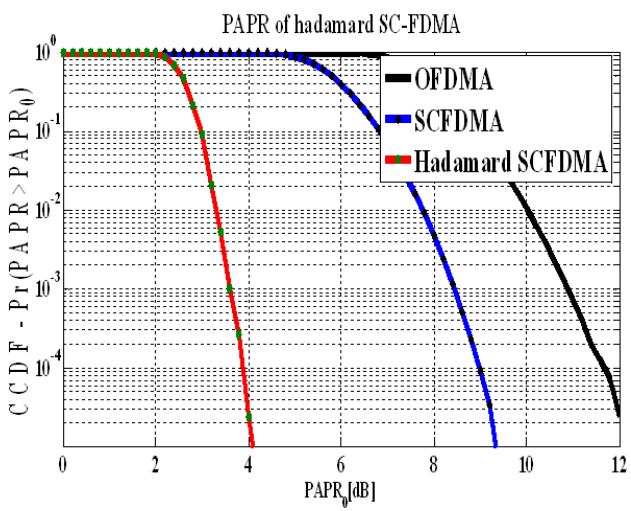

(b) With 16-QAM

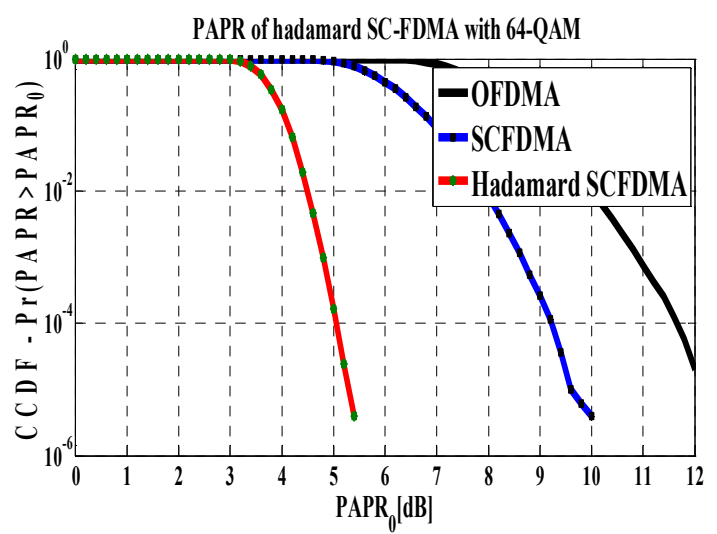

(c) With 64-QAM

Figure 5. Comparison of PAPR for OFDMA, SCFDMA and proposed scheme of hadamard SCFDMA

We can see in figure $5(\mathrm{~b})$ at $\mathrm{CCDF}=10^{-4}$, the PAPR of the proposed scheme is almost $5.1 \mathrm{~dB}$ smaller than that of classical SCFDMA scheme. And in figure 5(c) at $\mathrm{CCDF}=$ $10^{-4}$, the PAPR of the proposed scheme is almost $4.1 \mathrm{~dB}$ smaller than that of classical SCFDMA scheme. From the result show in figures and discuss of these results we can see the PAPR is varieties with the type of modulation order techniques, at the modulation order increase the reduction value of PAPR decrease.

In the other hand the BER rate is the most important parameter to evaluate this proposed scheme compared to the conventional SCFDMA. From Figure 6 we can see the BER is not degraded.

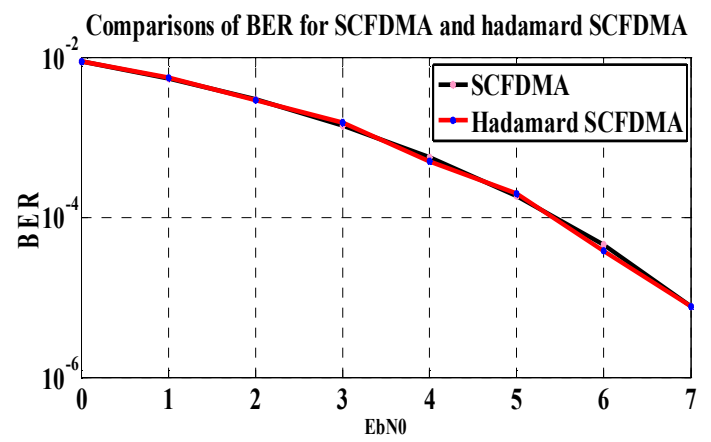

Figure 6. Comparisons of BER for SCFDMA and proposed scheme of Hadamard SCFDMA.

\section{Conclusion}

In this paper, a PAPR reduction scheme based on the Hadamard transform instead of FFT is proposed. Note that Hadamard transform is an orthogonal linear transform and can be implemented by a butterfly structure as in FFT. This means that applying Hadamard transform does not require the extensive increase of system complexity. The DFT spreading techniques of SCFDMA is based on Localized FDMA technique. The PAPR reduction performance and BER performance are evaluated by computer simulation. Simulation results state that the PAPR reduction performance is improved in the proposed techniques compared with conventional SCFDMA and is varied with the modulation order used as mapper techniques. On the other hand, the BER of system using proposed PAPR reduction scheme is not degraded and the complexity of the system is reduced.

\section{References}

[1] D. Falconer, et.al. ,"Frequency domain equalization for singlecarrier broadband wireless systems", IEEE Commun. Mag., 2002, 40, pp. 58-66.

[2] F. Adachi, et.al. ,"Broadband CDMA techniques", IEEE Wirel. Commun., 2005, 12, (2), pp. 8-18. 
[3] H.G. Myung, et.al. ,"Single carrier FDMA for uplink wireless transmission”, IEEE Veh. Technol. Mag., 2006, 1, (3), pp. 3038 .

[4] M. Vittal., et.al., "PAPR analysis of single carrier FDMA signals with pulse shaping", International conference on Communication and Signal Processing, April 3-5, 2013, India.

[5] Li X., Cimini L.J., "Effects of clipping and filtering on the performance of OFDM". Proc. IEEE Vehicular Technology Conf., May 1997, vol. 3, pp. 1634-1638.

[6] J. Armstrong., "Peak-to-average power reduction for OFDM by repeated clipping and frequency domain filtering", Electron. Lett., 2002, 38, pp. 246-247

[7] X. Li., Cimini L.J, "Effects of clipping and filtering on the performance of OFDM", IEEE Commun. Lett., 1998, 2, (5), pp. $131-133$

[8] H. G. Myung, "Introduction to Single Carrier FDMA," 15th European Signal Processing Conference (EUSIPCO) 2007, Poznan, Poland, Sep. 2007.

[9] H. G. Myung, et.al., "Peak-to-Average Power Ratio of Single Carrier FDMA Signals with Pulse Shaping", The 17th Annual IEEE International Symposium on Personal, Indoor and Mobile Radio Communications (PIMRC "06), Helsinki, Finland, Sep. 2006.

[10] M. Park, et.al., "PAPR reduction in OFDM transmission using Hadamard transform", IEEE International Conference o Communications, Vol.1, Jun 2000, pp.430-433. 